kennung der verletzten Person und ihrer Rechte im Strafverfahren. Schließlich war die Diskussion um die Opferrechte bei Schaffung der Reichsstrafprozessordnung weiter vorangeschritten als von den Verfechtern eines streng formalisierten Verfahrens angenommen ${ }^{35}$.

Dass der Gesetzgeber bislang kein Gesamtkonzept für die Einbettung der verletzten Person in das Strafverfahren gefunden hat, mag auch an den fehlenden Anregungen durch die deutsche Strafrechtswissenschaft liegen ${ }^{36}$. Da bereits im Rahmen der Ausbildung der zukünftigen Juristinnengeneration die verletzte Person mit ihren Rechten im Strafverfahren ins Abseits gerät, kann eine solche Anregung aus der Strafrechtswissenschaft in Zukunft auch nicht erwartet werden.

Susanne Wollmann (LL.M.) ist Wissenschaftliche Mitarbeiterin am Institut für Sanktionenrecht und Kriminologie Kiel.

\section{Fußnoten}

1 Der ausführliche Titel des Gesetzes lautet „Erstes Gesetz zur Verbesserung der Stellung des Verletzten im Strafverfahren", BGBl. I, S. 2496. Die Bezeichnung des Opfers in der Kurzfassung wurde erst aufgrund einer Entscheidung im Bundestag und in Anlehnung an den Fraktionsentwurf der SPD (BT-Drucks. 10/3636) aufgenommen, vgl. Rieß, JA 1987, 281 FN 8.

2 BT-Drucks. 10/5305, S. 8, 16.

3 So Haffke unter Verweis auf die Formalisierung der Strafrechtspflege und die Unschuldsvermutung, die für die beschuldigte Person gelte, KJ 2005, 17, 18.

4 Neuhaus sieht darin sogar die innere Struktur der Hauptverhandlung gefährdet, StV 2004, 620 626

5 BGBl I 2006 S. 3416 vom 30. Dezember 2006. Der ausführliche Titel lautet: „Zweites Gesetz zur Modernisierung der Justiz“.

6 Breits Hassemer merkt an, dass die verletzte Person in der Juristenausbildung nicht vorkommt, in Hassemer /Reemtsma, Verbrechensopfer - Gesetz und Gerechtigkeit, 2002, S. 29.

7 Rn. 4 ff.
8 Rn. 6f.

9 vgl. Ranft, Strafprozessrecht, 3. Auflage 2005, §1 Rn. 3f.

10 Rn. 602.

11 Rn. 603.

12 Der ausführliche Titel ist „Erstes Gesetz zur Modernisierung der Justiz“, BGBI. I 2004, S. 2198.

13 Dessen ausführlicher Name lautet "Gesetz zur Verbesserung der Rechte von Verletzten im Strafverfahren“, BGBl. I 2004, S. 1354.

14 Ab Rn. 430.

15 Vgl. Fall 51-52 auf S. 239f.

16 Rn. 605.

\section{Vgl. Rn. 337b.}

$18 \mathrm{Zu}$ diesem und ähnlichen Begriffen Jung, ZStW 93 (1981), 1147, 1148; Weigend, Deliktsopfer und Strafver-fahren (1989), S. 13.

19 Rn. 6ff, 11f. Dabei ist die Funktion des Strafprozesses „Schutz der verletzten Person" mit den anderen Aufgaben des Strafverfahrens in eine ausgewogene Balance zu bringen, vgl. Rn. 15 .

20 Rn. $525 \mathrm{ff}$.

21 Rn. 4.

22 Rn. 527ff, 531ff. Im Gegensatz hierzu bespricht Beulke das Recht der privaten Ermittlungen der verletzten Person allein bei den Beweisverwertungsverboten unter der Überschrift „Folgen rechtswidriger Erlangung von Beweismitteln durch Privatpersonen“, Rn. 478ff.

\section{Rn. 530.}

24 Zunächst im Rahmen des Ermittlungsverfahrens (Rn. 536f.) und dann bei der Behandlung des Hauptverfahrens (Rn. 735ff.). Wobei letztere Abhandlung wegen fehlender Überschrift im Inhaltsverzeichnis im Buch schwerer zu finden ist.

25 Rn. 738.

26 Vgl. BGHSt 49, 72, 79.

27 Vgl. BGHSt 49, 72, 82f.

28 Rn. 563.

29 Rn. $173 \mathrm{ff}$.

30 Vgl. auch Rn. 380f.

31 Wissenswertes zur Zeugenvernehmung findet sich in $\mathrm{Rn}$. 968ff. sowie $992 \mathrm{ff}$.

32 Das Argument der Einschränkung der Verteidigung wurde zur Zeit der Schaffung der RStPO von v. Forcade de Biaix vorgetragen, zitiert nach Güler, Die Opferposition im reformierten Strafverfahren in der zweiten Hälfte des 19. Jahrhunderts (2005), 23. Insbesondere Schünemann kritisierte bereits bei Einführung des Opferschutzgesetzes 1986 nicht nur die Möglichkeit der Nebenklage als Schwächung der Position der Verteidigung, NStZ 1986, 193, 198.

33 Zur Kritik hieran vgl. Höynck/Jesionek, MschrKrim 2006, 88, 103.

34 Zur Aufgabe des Verteidigers darauf zu achten, dass die rechtsstaatlichen Formalien im Straf-

verfahren eingehalten werden, vgl. Klemke/Elbs Rn. 34ff.

35 Vgl. hierzu Güler, Die Opferposition im reformierten Strafverfahren in der zweiten Hälfte des 19. Jahrhunderts (2005).

36 Zur Kritik am fehlenden Gesamtkonzept Höynck/Jesionek, MschrKrim 2006, 88, 90.

$37 \mathrm{Vgl}$. hierzu unter anderem mit dem Argument, dass funktionierende Systeme mit langer Tradition nur behutsam umgewandelt werden sollten Höynck, Das Opfer zwischen Parteirechten und Zeugenpflichten (2005), 211.

Frank Neubacher zur Studie von Roberto Saviano

\title{
Kriminologie der Camorra
}

$\mathrm{D}$ ies ist keine übliche Rezension - so wenig wie das $\mathrm{zu}$ besprechende Buch gewöhnlich ist oder sich als im eigentlichen Sinne wissenschaftlich bezeichnen ließe. Und doch beantwortet es Fragen, die nach der Lektüre wissenschaftlicher Werke offen bleiben. Wie lässt sich die Camorra, oder wie es in Neapel bei vielen heißt: "das System“, am besten aus der Sicht der Betroffenen beschreiben? Welchen Einfluss und welche Wirkung hat sie auf das alltägliche Leben und Empfinden der Neapolitaner? Oder auch: Warum schießen Camorristi nachts in die Schaufensterscheiben von Ladengeschäften? - Savianos Buch ist keine nüchterne wissenschaftliche Abhandlung. Es handelt sich um eine Mischung aus Reportage, Essay und Erzählung mit unübersehbaren stilistischen Qualitäten. Wortgewaltig und leidenschaftlich schildert, nein, klagt er das triste Leben in den von der Camorra heimgesuchten Quartieren Neapels und Umgebung an. Er beobachtet, analysiert, fragt sich durch, lässt nicht locker, und das an einem Ort, an dem schon die Verwendung des Wortes „Camorra" Missbilligung auslösen kann. Saviano hat, um rechtzeitig am Ort des Geschehens sein zu können, Polizeifunk gehört. Er hat zur Hochzeit des Camorra-Krieges im Winter 2004/2005 die Stadt auf seiner Vespa durchstreift, ist von der Polizei, den Carabinieri sowie von Kontrollposten der konfligierenden Clans gestoppt und durchsucht worden. Und er ist seiner Arbeit nachgegangen - als Journalist und Mitglied des „Osservatorio sulla camorra e l'illegalità", einer Initiative und Plattform der Tageszeitung „Corriere del Mezzogiorno".

Saviano hat sie also aus nächster Nähe gesehen - die ausgebrannten Autos mit den verkohlten Leichnamen, die verrenkten Glieder von Hingerichteten, das Leid und den Schmerz der Hinterbliebenen. Seine Sprache ist präzise, prägnant, seine Schilderungen drastisch, manchmal auch brutal. Keine Chance für Romantik à la „Bella Italia“ und „O sole mio", keine Chance für Bilder von Meer, Pizza, Zitronenbäumen. Saviano beschreibt die Farbe und den Geruch von Blut ebenso wie die verzweifelten Versuche der Opfer, den Todeskommandos $\mathrm{zu}$ entkommen. Er stellt gerade strafmündig gewordene Jugendliche vor, die davon träumen, Geschäfte, Frauen und Autos zu besitzen und „respektiert" zu werden, auch um den Preis eines frühen Todes, Jungen, die, von der Camorra mit einem Motorroller versorgt 
oder mit 200 Euro pro Woche alimentiert, im lukrativen Drogenhandel tätig sind oder in Schichten das Viertel überwachen, und auch Jungen, die darüber streiten, auf welche Weise sie getötet werden wollen, wenn es so weit ist. Wir erfahren: Schüsse in den Kopf werden von Halbwüchsigen, die schon $\mathrm{zu}$ viel gesehen haben, bevorzugt, weil sie einen schnellen Tod versprechen. Schüsse in die Brust hingegen führen dazu, dass sich die Lungen mit Blut füllen, dass sich der unglücklich Getroffene vor seinem Ableben in die Hosen macht.

In Italien hat dieser Erstling des 1979 in Neapel geborenen Autors eingeschlagen wie eine Bombe. Die einen rühmen ihn, sprechen davon, ein Schriftsteller sei geboren, andere haben vielleicht Gründe, die detaillierten Informationen sowie die ausgelöste Publicity zu fürchten. Die Polizei hat Saviano jedenfalls unter Polizeischutz gestellt, sein Aufenthaltsort ist unbekannt. Das aufwühlende Buch erscheint $\mathrm{zu}$ einem Zeitpunkt, in dem in- und ausländische Medien ihr Lager in Neapel bezogen haben. Schließlich kommt es auch in Italien nicht oft vor, dass Staatspräsident und Regierungschef, wie im November 2006 geschehen, ernsthaft einen Militäreinsatz erwägen, und zwar nicht in einem afrikanischem Krisengebiet, sondern vor der eigenen Haustür. Der Medienzirkus treibt allerdings seltsame Blüten, wie Saviano aus erster Hand $\mathrm{zu}$ berichten weiß. Die punktuelle Aufmerksamkeit lässt vor allem die wahren Dimensionen des Problems in Vergessenheit geraten. Saviano hat nachgezählt: 3.600 von der Camorra getötete Menschen seit 1979 - mehr als die Mafia auf Sizilien oder die 'Ndrangheta in Kalabrien auf dem Gewissen haben. Mit Ausnahme der Jahre 1999 (91), 2001 (80), 2002 (63), 2003 (83) und 2005 (90) waren es nie weniger als 100 Tote pro Jahr, zeitweise auch über 200. Und: Die „militärischen" Operationen der Camorra lenken die Öffentlichkeit von ihrem ökonomischen Erfolg und ihrer politischen Einflussnahme ab. Seit 1991 wurden in Kampanien 71 Gemeinderäte (in Worten: einundsiebzig) wegen Infiltration durch die Camorra aufgelöst und vorübergehend der Leitung durch einen Staatskommissar unterstellt. Ohne dass traditionelle Einnahmequellen aus Schutzgelderpressung, Waffenhandel oder Kartellen versiegt wären, haben der Schmuggel von Massenprodukten des Alltags und die Produktpiraterie den Zigarettenschmuggel in seiner Bedeutung abgelöst und stellen neben dem Drogenhandel das zweite wichtige Standbein der Camorra dar. Sportschuhe, Plastikspielzeug, Videospiele, besonders aber Stoffe und Textilien werden in großem Stil eingeführt, am Zoll vorbeigeschafft und mit Gewinn verkauft. Saviano schreibt von Fabriken der einzelnen Clans, wo Lederjacken, Anzüge und Kostüme in Schwarzarbeit produziert und mit den begehrten Etiketten italienischer Modemacher versehen werden, um kurz darauf in Läden europäischer Großstädte zu landen, die die Camorra mit Hilfe von Strohleuten installiert hat. Und an „schwarzen“ Arbeitskräften herrscht in manchen Gemeinden im Norden Neapels, in denen die Arbeitslosenquote an die $40 \%$ heranreicht, kein Mangel.

Warum Camorristi auf Schaufensterscheiben schießen? Nicht immer, um den Ladeninhaber einzuschüchtern. Vielleicht probieren sie einfach neu erworbene Kalaschnikows aus. Oder sie haben einen "Freund", der Glaser ist. Und auch wenn es keinen Grund gibt - der Ladeninhaber wird es selbst am besten wissen, denn einen Grund für ein schlechtes Gewissen gibt es immer.

Gomorra - das ist der Haupttitel des Buches und der Name jener alttestamentlichen Stadt, die Gott wegen ihrer Sünden mit einem Tod bringenden Regen aus Feuer und Schwefel bestrafte. Napoli - das ist für Saviano ein Fegefeuer, Sinnbild für Tod und Illegalität, Ort entwürdigender Lebensumstände, gegen die er revoltieren muss. Im Schlusssatz schleudert er dem Leser, Italien, Neapel und dem System seine Wut und seinen ohnmächtigen Überlebens- willen entgegen: „Maledetti bastardi, sono ancora vivo!" (Ihr verfluchten Bastarde, noch bin ich lebendig!) - Worte, die gewiss nicht als provokative Aufforderung zur Tötung zu verstehen sind, sondern als Selbstvergewisserung desjenigen, der Schreiben zu seiner Überlebensstrategie erwählt hat und nun die Bilder von Tod, Verwesung und Unrat, die er in sich aufgenommen hat, hochwürgt und ausspuckt, um nicht daran zu ersticken.

Wer etwas über den langen Schatten erfahren will, den die Camorra auf das Leben in Neapel und Kampanien wirft, und über das Zwielicht, in das sie den scheinbar ganz normalen Alltag taucht, der lese das Buch von Saviano. Es erscheint demnächst in deutscher Sprache.

Professor Dr. Frank Neubacher M.A., Friedrich-Schiller-Universität Jena.

Saviano, Roberto: Gomorra, Viaggio nell'impero economico e nel sogno di dominio della camorra, Arnoldo Mondadori Editore, Milano 2006, 331 Seiten 15,50 Euro

Kirstin Drenkhahn zu Ulrich Sieber und H.J. Albrecht

\section{Strafrecht und Kriminologie unter einem Dach}

B ei dem von Ulrich Sieber und Hans-Jörg Albrecht herausgegebenen Band handelt es sich zwar um ein Geschenk für Hans-Heinrich Jescheck zum 90. Geburtstag im Januar 2005, es soll aber keine typische juristische Festgabe sein. Das Buch erschien nicht zum Geburtstag selbst, sondern ungefähr ein Jahr später und dokumentiert das eigentliche Geschenk, nämlich ein Kolloquium zum Thema „Kriminologie und Strafrecht unter einem Dach".

Am Anfang des Buches wie des Kolloquiums stehen Glückwünsche von Vertretern einiger Institutionen, denen Jescheck angehört hat und teilweise auch noch heute angehört ( $\mathrm{S}$. 3-27). Auf die Begrüßung durch Ulrich Sieber, gemeinsam mit seinem Ko-Herausgeber HansJörg Albrecht, Direktor des Freiburger Max-Planck-Instituts für ausländisches und internationales Strafrecht, folgen der badenwürttembergische Justizminister Ulrich Goll und der damalige Leiter der Strafrechtsabteilung im Bundesjustizministerium Peter Wilkitzki. Daran schließen sich Glückwünsche der AlbertLudwigs-Universität Freiburg durch den Rektor Wolfgang Jäger und der Rechtswissenschaftlichen Fakultät der Universität Freiburg durch den damaligen Dekan Andreas Voßkuhle an.
Darüber hinaus gratulierten für die Association Internationale de Droit Pénal deren Präsident José Luis de la Cuesta, für die Gesellschaft für Rechtsvergleichung der damalige Vorsitzende des Vorstands Uwe Blaurock, für die Max-Planck-Gesellschaft deren ehemaliger Präsident Hans M. Zacher sowie für die Société Internationale de Défense Sociale deren Vorstandsmitglied Klaus Tiedemann, der Jescheck zudem die ihm von der Société verliehene Cesare Beccaria-Medaille überreichte. In ihren kurzen Glückwunschadressen würdigen die Gratulanten Jeschecks herausragende Leistungen im Strafrecht und berichten aus seinem Leben, 